\title{
On the solar poloidal magnetic field as one of the main factors for maximum GCR intensity for the last five sunspot minima
}

\section{M.B. Krainev, ${ }^{a, *}$ B.B. Gvozdevsky, ${ }^{b}$ M.S. Kalinin, ${ }^{a}$ O.P.M. Aslam, ${ }^{c}$ M.D. Ngobeni ${ }^{c}, d$ and M.S. Potgieter ${ }^{e}$}

${ }^{a}$ Lebedev Physical Institute, Russian Academy of Sciences, 119991 Moscow, Russia

${ }^{b}$ Polar Geophysical Institute, Russian Academy of Sciences, 184209 Apatity, Russia

${ }^{c}$ Centre for Space Research, North-West University, 2520 Potchefstroom, South Africa

${ }^{d}$ School of Physical and Chemical Sciences, North-West University, 2745 Mmabatho, South Africa

${ }^{e}$ Institute for Experimental and Applied Physics, Christian Albrechts University in Kiel, 24118 Kiel, Germany

E-mail: kalinin273@sci.lebedev.ru, gvozdevsky@pgia.ru, mkrainev46@mail.ru, aslamklr2003@gmail.com, Donald.Ngobeni@nwu.ac.za,

Marius.S.Potgieter@gmail .com

\begin{abstract}
The conditions in the heliosphere are considered during the minimum phase of the sunspot cycle when the intensity of galactic cosmic rays (GCRs) attains its maximum at the Earth. These times of maximum GCR intensity are determined for the last five sunspot minima, including the present one. From the quantitative correlation between the heliospheric factors important to the modulation of GCRs in the heliosphere and the index of high-latitude photospheric magnetic field (all determined corresponding to times of GCR intensity maxima) the conclusion is made that the poloidal magnetic field of the Sun is one of the main governing factors for these heliospheric characteristics.

Following this up, the dependence of proton spectra near the Earth on the index as mentioned above for the last five sunspot minima, $21 / 22$ to $24 / 25$, is calculated, also taking into account the strength of the heliospheric magnetic field and the tilt of the heliospheric current sheet, the solar wind speed and the position of the termination shock as the observable factors depending on the high-latitude photospheric magnetic field. The calculations are discussed with special attention paid to the comparison of spectra for the current and previous sunspot minima. The conclusion is made on the general dependence of GCR spectra on the poloidal magnetic field of the Sun.
\end{abstract}

$37^{\text {th }}$ International Cosmic Ray Conference (ICRC 2021)

July 12th - 23rd, 2021

Online - Berlin, Germany

\footnotetext{
*Presenter
} 


\section{Introduction}

In this paper we continue a quantitatve study of the concept that the poloidal branch of solar activity is very important for heliospheric processes, including GCR modulation, during the periods of medium and low sunspot activity. For our previous work on this subject, see Krainev [5, 6], Krainev et al. [7]. Here, we further explore the dependence of the maximum GCR intensity on the index of high-latitude photospheric solar magnetic field (SMF) during the last five sunspot minima using numerical calculations of steady-state 3D models which we have started to explore as reported by Krainev et al. [7]. The GCR observations in a wider energy range will be considered, the list of the observable factors determining the calculated intensity will be extended, and most important, all these factors will be considered as a function of the high-latitude photospheric SMF. This means that the dependence of GCR spectra directly on the poloidal SMF will be investigated.

First, we consider GCR observations near the Earth to determine the times of maximum GCR intensity $\left(t_{J}^{\max }\right)$ during the last five sunspot minima including the present one, 24/25, and the dependence of the observed main heliospheric (HS) factors of importance to GCR modulation on the index of the high-latitude SMF, all corresponding to the determined $t_{J}^{\max }$. Then we calculate and discuss the proton spectra near the Earth both for actual values of the above main HS factors during the last two sunspot minima and how they change along trends of linear dependence of the above mentioned HS factors on the high-latitude SMF.

\section{Observations: maxima of GCR intensity, heliospheric factors and SMF}

Fig. 1 shows the time profiles of the monthly averaged GCR intensity from 1970 to 2020 at the Earth for three long-term observational series which are sensitive to different energies. The data are normalized to $100 \%$ for 1997 which was the previous sunspot minimum with the HMF polarity as $A>0$. When compared to Krainev et al. [7] we added the kinetic energy range $T<100$ $\mathrm{MeV}$ to make the situation at low energies clearer. The times of maximum GCR intensity $\left(t_{J}^{\max }=\right.$ $1977.4,1987.15,1997.40,2009.62,2020.3)$ for all five sunspot minima are shown by vertical lines in Fig. 1. Uncertainty of these times is around 1 to 2 months because of the weak dependence on the energy. In the present sunspot minimum (24/25, April-May 2020) for low energies (Fig. 1 a, b) the level in 2009 has been surely surpassed already (by $\Delta \approx 17.1 \%$ for $T=54-76 \mathrm{Mev} / \mathrm{n}$ carbon and $\Delta \approx 6.8 \%$ for $T=200 \mathrm{MeV}$ protons), but for intermediate and higher energies (Fig. $1 \mathrm{c}, \mathrm{d}$ ) it has not been attained yet (except the Moscow and some other neutron monitors). For the regular balloon monitoring $(\mathrm{RBM})$ data, the proton integral intensity $J(T>100 \mathrm{MeV}), \Delta \approx-0.96, \%$ and for Hermanus NM, $\Delta \approx-0.46 \%$.

As shown by Krainev et al. [7] the values of the HS factors, corresponding to maximum GCR intensity, in the first approximation vary according to the index of the average high-latitude SMF, $B_{l s}^{p o l}=\left(\left|B_{l s}^{N}\right|+\left|B_{l s}^{S}\right|\right) / 2$, where $B_{l s}^{N}$ and $B_{l s}^{S}$ are the line-of-sight components of the high-latitude photospheric SMF in the N- and S-hemispheres of the Sun (WSO Site [25]). The four upper panels of Fig. 2 show the HS factors corresponding to the maximum GCR intensity as a function of $B_{l s}^{p o l}$; the linear trend calculated as a least square fit to all sunspot minima is shown by the tilted straight lines in each panel. The lowest panel will be discussed later. 


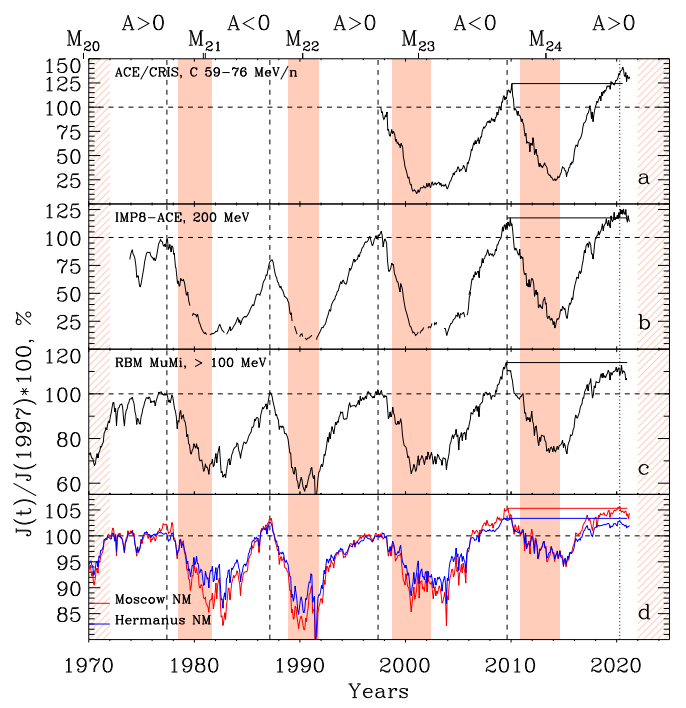

Figure 1: Time profiles of GCR intensity, normalized to $100 \%$ in 1997, for the period 1970 to 2020 . Periods of SMF polarity reversals are shown by the vertical shaded bands (Krainev [6]). Times of maxima in the sunspot cycle and the dominant SMF polarity, $A$, are indicated at the top. Vertical dashed lines show the times of maximum intensity during minima 21/22, 22/23, 23/24, whereas the dotted line is for minimum 24/25. Horizontal lines mark the maximum levels attained in minimum 23/24. Here, the GCR carbon intensity is shown for lowest energies (panel a, $T=54-76 \mathrm{MeV} / \mathrm{n}$ CRIS/ACE Site [23]), while proton intensity is shown for low (panel b, $T=200 \mathrm{MeV}$, McDonald [10], CRIS/ACE Site [23]), intermediate (panel c, RBM experiment, Stozhkov et al. [18], LPI/RBM Site [21]) and high energies (panel d, neutron monitors, Moscow IZMIRAN/CR Site [20], black; Hermanus SA NM Site [22], blue).

Note the pronounced linear dependence on $B_{l s}^{p o l}$ for all HS factors and minima (probably, with exception of all factors for minimum 20/21 and $\alpha_{t}$ for 24/25). In particular, $B, V$, and $N V^{2}$ were increasing monotonically with increasing $B_{l s}^{p o l}$, while $\alpha_{t}$ had decreased. Similar to Krainev et al. [7] and in accordance with our notion of the importance of the poloidal SMF for the processes in the heliosphere during low and medium sunspot activity, we consider the changes in the strength of the poloidal SMF field as the main cause of changes in the HS factors important to GCR modulation and corresponding to $t_{J}^{\max }$.

\section{Calculations: Proton spectra for last two minima and trend with SMF}

Following Parker [12], Krymskiy [9], Parker [13] and using the phase-space distribution function $f(\vec{r}, p, t)=J(\vec{r}, T, t) / p^{2}$ instead of GCR intensity $J(\vec{r}, T, t)$, where $p$ is the momentum of particles, the Fokker-Plank transport equation (TPE) for the steady-state case is as follows:

$$
\nabla \cdot\left(K^{s} \cdot \nabla f\right)-\vec{V} \cdot \nabla f+\frac{\nabla \cdot \vec{V}}{3} p \frac{\partial f}{\partial p}-\vec{V}^{d r} \cdot \nabla f=0
$$

where $\vec{V}, \vec{V}^{d r}$ and $K^{s}$ are the solar wind and particle drift velocities and the symmetric (diffusion) part of the propagation tensor, respectively. The boundary (in a heliocentric spherical system of 


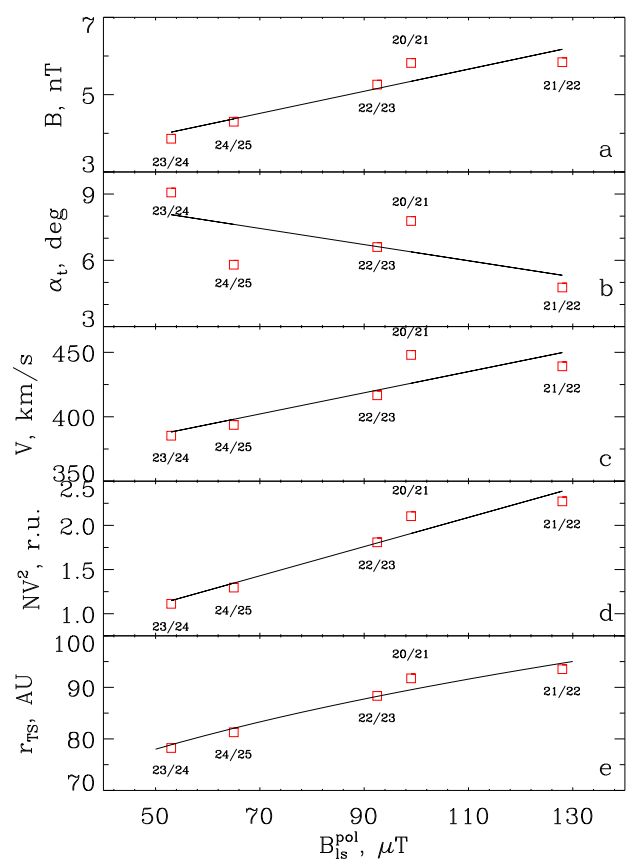

Figure 2: The main HS factors corresponding to maximum GCR intensity for the last five sunspot minima as a function of the magnitude of the poloidal magnetic field of the Sun. The HS factors shown in the panels are: (a) The HMF magnitude at the Earth OMNI Site [24]; (b) The tilt angle of the HCS $\alpha_{t}$ WSO Site [25]; (c, d) The SW speed $V$ and ram pressure $N V^{2}$ OMNI Site [24]; (e) the radial distance from the Sun to the termination shock $r_{T S}$ determined as discussed in the next section. The symbols for various sunspot minima mark the real values corresponding to $t_{J}^{\max }$, while the solid tilted lines are for a linear trend calculated as a least square fit for all sunspot minima.

coordinates) and initial conditions (at $p=p_{\max }$ ) are given as follows:

$$
\begin{array}{ll}
\left.\frac{\partial f}{\partial r}\right|_{r=r_{\text {min }}} & =0,\left.\quad f\right|_{r=r_{\text {max }}}=f_{n m}(p),\left.\quad \frac{\partial f}{\partial \vartheta}\right|_{\vartheta=0, \pi}=0 \\
\left.f\right|_{p=p_{\text {max }}} & =f_{\text {nm }}\left(p_{\text {max }}\right),
\end{array}
$$

where $r_{\min }, r_{\max }, p_{\max }$ are, respectively, the radii of the inner and outer boundaries of the modulation region and the momentum above which there is no modulation and where $f_{n m}(p)$ is the distribution function corresponding to the very local interstellar spectrum Bisschoff et al. [4]. The coefficients of the TPE are modeled according to the HS factors assumed important to GCR modulation, and the processes of their interaction with charged particles (see Potgieter [14, 15]). For calculating the proton intensity in the sunspot minima and its dependence on the high-latitude SMF, the full three-dimensional (3D) model and code for the TPE coefficients described by Aslam et al. [2,3] and by Potgieter and Vos [16] are used including a set of observable parameters, $S_{o b s}$, and a set of other (descriptive) parameters, $S_{\text {descr }}$, which are required for a convincing description of GCR observations. 
Unlike Krainev et al. [7], where the set of odservable parameters includes only $B, \alpha_{t}, A$, we included in $S_{o b s}$ all HS factors as shown in Fig. 2. This means that the solar wind velocity $V$ and ram pressure $N V^{2}$, as well as $B$ and $\alpha_{t}$, all showing the trend with $B_{l s}^{p o l}$, are included in $S_{o b s}$. The global model of solar wind velocity (e.g., Potgieter [14]), describes the latitudinal transition of the velocity from the equatorial (low) value $V_{e q} \approx 430 \mathrm{~km} / \mathrm{s}$ to the polar (high) value $V_{\text {pol }} \approx 750 \mathrm{~km} / \mathrm{s}$. We set $V_{e q}$ equal to the velocity $V$ observed near the Earth and corresponding to maximum GCR intensity. For the solar wind ram pressure $N V^{2}$, we take into account the correlation between this HS factor and the position $r_{T S}$ of the solar wind termination shock (TS). Here, we use the relation $r_{T S}=r_{T S, 0} \cdot\left(N V^{2} / N V_{0}^{2}\right)^{\alpha}$. Using this relation with $r_{T S, 0}=78$ AU for 2009 and $\alpha=0.25$, the position of TS will approximately satisfy its time dependence inferred by Richardson and Wang [17] (see their Fig. 1). The results for the radial distance from the Sun to the TS position $r_{T S}$ for five sunspot minima and for the linear trend of $N V^{2}$ as a function of $B_{l s}^{p o l}$, are shown in panel (e) of Fig. 2. See also Vos and Potgieter [19].

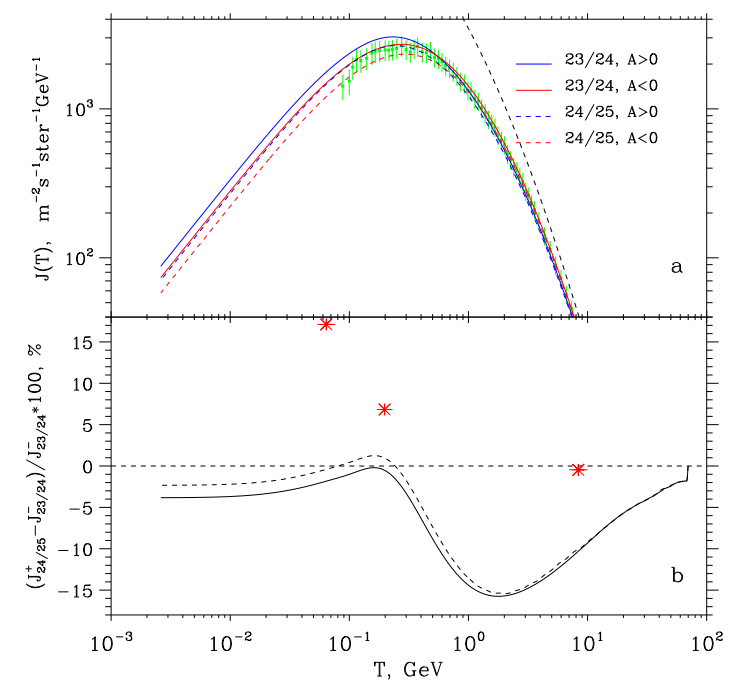

Figure 3: Panel a: Calculated spectra for GCR protons at the Earth for sunspot minima 23/24 to 24/25. Short vertical dashes are for the proton spectrum measured by PAMELA during minimum 23/24; November, 2009; $A<0$ Adriani et al. [1]. Our calculated spectra are shown for minima 23/24 (solid) and 24/25 (dashed lines) for both types of HMF polarity; red line is for the $A<0$ and blue line for the $A>0$ polarity cycle. The black dashed line is the local interstellar proton spectrum according to Bisschoff et al. [4]. Panel b: Difference between calculated spectra for minima 24/25 $(A>0)$ and 23/24 $(A<0)$ calculated in this paper (solid line) and in Krainev et al. [7] (dashed line). The observations of the difference between the spectra for the current and previous sunspot minima (see discussion in the previous section) are shown as red stars.

Fig. 3 (a) shows the calculated proton spectra for sunspot minima 20/21 to 24/25 for both types of HMF polarity as well as the proton spectrum measured by the PAMELA detector at the Earth in minimum 23/24, for November 2009, $A<0$, Adriani et al. [1]. All spectra were calculated using the sets of observable parameters $\left(B, \alpha_{t}, V, r_{T S}\right)$ corresponding to both minima and shown by the specific symbols in Fig. 2 but the same (except $V_{e q}$ and $r_{T S}$ ) set of the descriptive parameters $\left(S_{d e s c r}^{23 / 24}\right)$ used by Ngobeni et al. [11] for describing the proton spectrum in sunspot minimum 23/24. 
It is easily seen that for each minimum the red and blue lines for spectra of different polarity intersect producing a clear spectral cross-over at $T_{c o}$. The change of $T_{c o}$ as well as other features of the spectra will be shown and discussed later.

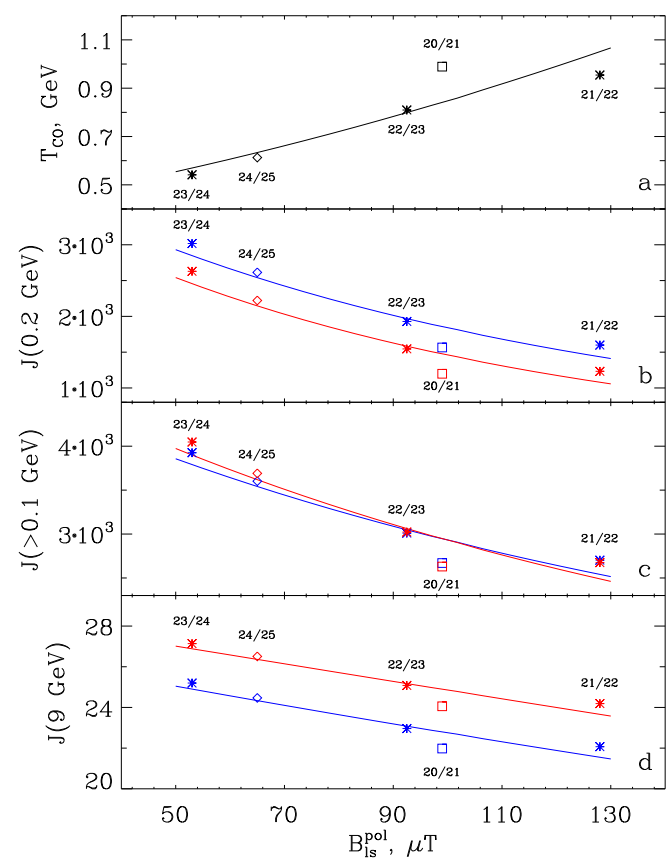

Figure 4: The dependence of the calculated GCR features at the Earth on $B_{l s}^{p o l}$ under the condition of a linear relation between $S_{o b s}=\left\{B, \alpha_{t}, V, N V^{2}\right\}$ and $B_{l s}^{p o l}$ (solid lines) and for the real set $S_{o b s}$ for each sunspot minimum (the same symbols as in Fig. 2). In panel (a) the dependence on $B_{l s}^{\text {pol }}$ of the corresponding spectral cross-over energy $T_{c o}$ is shown. Panels (b, c, d) show the dependence on $B_{l s}^{p o l}$ of the calculated proton intensity of low $J(T=200 \mathrm{MeV})$, intermediate $J(T>0.1 \mathrm{GeV})$ and high $J(T=9 \mathrm{GeV})$ energies, respectively, for both HMF polarities with the blue line for $A>0$ and red line for $A<0$.

Evidently, the calculated proton spectrum for minimum 23/24 with $A<0$, describes the 2009 observations very well. Since the comparison of GCR modulation in the two last sunspot minima is of particular interest for this work, note that the proton spectrum for the present minimum 24/25, calculated with $A>0$ (blue dashed line), practically coincides with that for the previous minimum 23/24 with $A<0$ (red solid line), whereas the illustrative $A<0$ spectrum for the present minimum (red dashed line) is lower than both spectra for minimum 23/24 (red and blue solid lines). The scale of Fig. 3 (a) is not fine enough to compare the calculated spectra for minima 25/24 (2020) and 23/24 (2009) with real HMF polarities, so that the relative difference between them, $\delta_{2020-2009}(T)=\left(J_{24 / 25}^{+}-J_{23 / 24}^{-}\right) / J_{23 / 24}^{-} * 100, \%$, with superscript index indicating HMF polarity, is calculated and shown by the solid line in panel (b) of the same figure. Evidently, this difference is negative for all energies although at $T \approx 0.2 \mathrm{GeV}$ it is very small. To relate this result with that in Krainev et al. [7], where the set of observable parameters included only $V, B, \alpha_{t}$, A, we show the difference, $\delta_{2020-2009}(T)$, calculated in Krainev et al. [7], by the dashed line in Fig. 3 (b). It 
can easily be seen that the change of the relative difference between the spectra for minima $24 / 25$ and $23 / 24$ caused by the extension of the set of observable parameters are rather small $(<2 \%)$. To illustrate how our calculations differ from observations of the difference between the spectra for the current and previous sunspot minima, the observed difference is shown in Fig. 3 (b) by the red stars based on indications in Fig. 1 and discussed afterwards.

The detailed changes in the energy spectra for both types of HMF polarity caused by the linear correlation of the observable HS parameters $B, \alpha_{t}, V, N V^{2}$ with increasing $B_{l s}^{p o l}$, are shown in Fig. 4. If we compare quantitatively the calculations with the extended set $S_{o b s}$ with those from calculations in Krainev et al. [7] with the limited set $S_{\text {obs }}$, the result are approximately the same (within $\approx 2 \%$ ). At the same time the scatter of the data for individual sunspot minima around the trend for the case of direct dependence of all HS factors on $B_{l s}^{p o l}$ is much stronger. Both of these facts reflect the much stronger dependence of the calculated spectra on $B$ when compared with other HS factors for sunspot minima. The dependence on $B, \alpha_{t}(B)$ of the calculated intensity of intermediate energies (or, rather, the integral intensity $J(T>0.1 \mathrm{GeV})$ ), which can be estimated from the RBM experiment (Stozhkov et al. [18], Krainev [8], LPI/RBM Site [21]), is shown in Fig. 4 (c) for both polarity types of the HMF and it follows that the curves are very close to each other, with $-0.015<\delta_{+/-}<0.011$ in the full range of $0.050<B_{l_{s}}^{p o l}<0.130 n T$.

\section{Conclusions}

For the present minimum $24 / 25$, the GCR intensity apparently has attained already a maximum value for the series shown in Fig. 1. Evidently, the 50-70 and $200 \mathrm{MeV}$ data for the present minimum is higher than in 2009; at intermediate energy and at NM energies it is somewhat lower, although to be conclusive, data from AMS02 for 2020 and 2021 will have to be added when available.

The close linear relation between the high-latitude SMF and different characteristics in the heliosphere, including those of the SW, HMF and GCR intensity, quantitatively describes the trend observed in the time dependence during all sunspot minima 20/21 to 24/25 and emphasizes the leading role of the poloidal branch of solar activity during minimum periods of the sunspot cycle.

The enhanced scatter of the data for individual sunspot minima around the trend for the case of direct dependence of all HS factors (including the solar wind speed and ram pressure) on $B_{l s}^{p o l}$ reflects the much stronger dependence of the calculated spectra on the magnitude of the HMF near the Earth $B$ when compared with other HS factors.

Our calculations of proton spectra for minima 23/24 and 24/25 and the trend of the spectra with high-latitude SMF, $B_{l s}^{\text {pol }}$, with the extended set of observable parameters, including all of them linear correlated with $B_{l s}^{\text {pol }}$, show approximately the same results (within 2\%) as our calculations with the limited set (without local solar wind velocity and ram pressure). It also reflects the leading role of HMF strength near the Earth $B$ among the other HS factors in the calculated GCR intensity in the periods of minimum sunspot activity.

MBK acknowledges the partial support of RFBR-NRF grant 19-52-60003 SA-t. MDN thanks the South African NRF for partial financial support under the Joint Science and Technology Research Collaboration between SA and Russia (grant no: 118915). 


\section{References}

[1] Adriani, O., Barbarino, G.C., Bazilevskaya, G.A., et al. Astrophys. J. 765, 91, 1-8, 2013.

[2] Aslam, O.P.M., Bisschoff, D., Potgieter M.S, et al. Astrophys. J. 873, 70, 1-11, 2019.

[3] Aslam O.P.M., Bisschoff D., Ngobeni M.D., et al. Astrophys. J. 909, 215, 1-13, 2021.

[4] Bisschoff D., Potgieter M.S., and Aslam O.P.M., Astrophys. J. 878, 59, 1-8, 2019.

[5] Krainev, M.B., Bull. Russian Academy Sci.: Phys. Allerton Press Inc. 81, 2, 166-169, 2017.

[6] Krainev, M.B., Solar-Terres. Phys. 5, 10-20, 2019.

[7] Krainev, M., Kalinin, M., Aslam, O.P.M., et al. Adv. Space Res. in press 2021.

[8] Krainev, M.B., Phys. Auroral Phen. Proc. XLII Ann. Sem. Apatity 130-133, 2019.

[9] Krymskiy, G.F., Geomag. Aeron. 4, 763-769, 1964.

[10] McDonald, F.B., Space Sci. Rev. 83, 33-50, 1998.

[11] Ngobeni, M.D., Aslam O.P.M,. Bisschoff D., et al. Astrophys. Space Sci. 365, 182, 2020.

[12] Parker, E.N., Phys. Rev. 110, 1445, 1958.

[13] Parker, E.N., Planet. Space Sci. 13, 9-49, 1965.

[14] Potgieter, M.S., Living Rev. Solar Phys. 10, 3-66, 2013.

[15] Potgieter, M.S., Adv. Space Res. 60, 848-864, 2017.

[16] Potgieter, M.S., and Vos, E.E., Astron. Astrophys. 601, A23, 1-10, 2017.

[17] Richardson, J.D., and Wang,C., Astrophys.J. Lett. 734, L21, 2011.

[18] Stozhkov, Yu.I., Svirzhevsky, N.S., Bazilevskaya, G.A., et al. Preprint 14, Lebedev Physical Institute, Moscow 77, 2007.

[19] Vos, E.E., and Potgieter M.S., Solar Phys. 291, 181-195, 2016.

[20] http://cr0.izmiran.ru/mosc/main.htm

[21] http://sites.lebedev.ru/en/sites/DNS_FIAN/1949.html

[22] http://natural-sciences.nwu.ac.za/neutron-monitor-data/

[23] http://www.srl.caltech.edu/ACE/CRIS_SIS/ cris.html|

[24] ftp://omniweb.gsfc.nasa.gov/pub/data/omni

[25] http://wso.stanford.edu/ 